\title{
Formulation of Model Defects Suitable for the Resonance Regime
}

\author{
Benedikt Raab $^{1, *}$, Thomas Srdinko ${ }^{1, * *}$, and Helmut Leeb $^{1, * * *}$ \\ ${ }^{1}$ Atominstitut, TU Wien, Wiedner Hauptstrasse 8-10, 1040 Vienna, Austria
}

\begin{abstract}
A method to account for model deficiencies in nuclear data evaluations in the resonance regime is proposed. The method follows the ideas of Schnabel and coworkers and relies on Gaussian processes with a novel problemadapted ansatz for the covariance matrix of model uncertainties extending the formalism to the energy region of resonances. The method was used to evaluate a set of schematic but realistic neutron reaction data generated by an R-matrix code and a well defined model defect. Using the extended ansatz for model defects the Bayesian evaluation successfully recovered the built-in model defect in size and structure thus demonstrating the applicability of the method.
\end{abstract}

\section{Introduction}

The availability of reliable evaluated nuclear data is an important prerequisite for developments in several fields of science and technology. At present, Bayesian evaluation techniques are considered as the proper tool to consistently combine experimental data with model information. Because of the complexity of nuclear systems, nuclear models can only describe certain properties of the system, but not the complete system. Consequently there exist always model defects and they have an important impact on evaluations [1,2]. Several attempts for their inclusion were proposed in the past [3,4]. Recently Schnabel [1] presented a statistically consistent formulation in terms of Gaussian processes, which was applicable for smooth energy dependence of reaction cross sections and more recently for angle differential cross sections [5].

In this contribution we propose an extension of the formalism of model defects for the resonance regime, especially suited for light nuclear systems. The essential step, presented in Sect. 2, is the formulation of a new ansatz for the covariance matrix of the model defects and its inclusion in the evaluation process. In Sect. 3 the schematic example, the details of the evaluation procedure and the results are presented and discussed. The work is summarized in Sect. 4 and an outlook is given.

\footnotetext{
*e-mail: benedikt.raab@tuwien.ac.at

**e-mail: thomas.srdinko@tuwien.ac.at

***e-mail: helmut.leeb@tuwien.ac.at
} 


\section{Evaluation including Model Defects}

\subsection{Concept of Formalism}

The mathematical basis of up-to-date evaluation techniques is provided by Bayes theorem

$$
\pi\left(\vec{p} \mid \vec{\sigma}_{\exp }\right)=\frac{1}{\int \mathrm{d}^{d} p l\left(\vec{\sigma}_{\exp } \mid \vec{p}\right) \pi(\vec{p})} l\left(\vec{\sigma}_{\exp } \mid \vec{p}\right) \pi(\vec{p}) .
$$

Thereby the $a$-priori distribution $\pi(\vec{p})$ of model parameters $\vec{p}$ is updated with experimental information represented by the likelihood function $l\left(\vec{\sigma}_{\exp } \mid \vec{p}\right)$, where $\vec{\sigma}_{\exp }$ is the vector of experimental data points. The fraction in Eq. (1) acts as normalization. The result is an $a$ posteriori distribution $\pi\left(\vec{p} \mid \vec{\sigma}_{\exp }\right)$ of model parameters taking into account the experiments.

In standard Bayesian evaluations it is assumed that the model is perfect, i.e. $\vec{\sigma}_{\text {exp }}=\vec{\sigma}_{\text {mod }}+\vec{\epsilon}_{\text {exp }}$, where $\vec{\sigma}_{\text {mod }}$ and $\vec{\epsilon}_{\text {exp }}$ are the vectors of model values and experimental errors, respectively. In general model defects always occur and their neglect affects the evaluation $[1,2]$. At present it is well established that model defects must be taken into account in nuclear data evaluation. There have been several attempts to account for model defects. Recently Schnabel [1, 5] developed a statistically well defined approach based on Gaussian processes. The evaluation starts from the ansatz

$$
\vec{\sigma}_{\text {exp }}=\vec{\sigma}_{\text {mod }}+\vec{\epsilon}_{\text {mod }}+\vec{\epsilon}_{\exp }
$$

where $\vec{\epsilon}_{\text {mod }}$ is the model defect. All quantities are assumed to be random variables. For simplicity we assume multivariate normal distributions, i.e.

$$
\vec{\sigma}_{\text {mod }} \sim \mathcal{N}\left(\vec{\sigma}_{0}, \mathbf{A}_{0}\right), \quad \vec{\epsilon}_{\text {mod }} \sim \mathcal{N}\left(\overrightarrow{0}, \mathbf{K}_{0}\right), \quad \vec{\epsilon}_{\text {exp }} \sim \mathcal{N}(\overrightarrow{0}, \mathbf{B}),
$$

where the first argument refers to the mean value and the second one to the associated covariance matrix. In addition we assume that $\vec{\sigma}_{\text {mod }}, \vec{\epsilon}_{\text {mod }}$ and $\vec{\epsilon}_{\text {exp }}$ are mutually independent.

\subsection{Model defects for the Resonance Regime}

The inclusion of model defects via a Gaussian process assumes a vanishing $a$-priori mean value of $\vec{\epsilon}_{\text {mod }}$ and an $a$-priori covariance matrix $\mathbf{K}_{0}$ which must be chosen according to the expectation. The covariance function for angle-integrated and angle-differential cross sections introduced in [1] and [5], respectively, is not suited for the resonance region. Frequently phenomenological R-matrix descriptions of the resonant part are considered to be perfect. However, the achievable description depends on several quantities which are chosen prior to a fit, i.e. the chosen matching radii, the number of poles, the choice of background poles etc. Of course one can consider these quantities as parameters of the fit, but high correlations and the strong non-linear relation to the observables will hamper reliable estimates of their uncertainties. Therefore a treatment as model defects of a specifically chosen model is preferable.

In order to account for model defects of the cross section $\sigma(E)$ in the resonance regime we propose for model defects the following covariance matrix,

$$
\begin{aligned}
\mathbf{K}_{0}\left(E_{i}, E_{j}\right)= & \sigma\left(E_{i}\right) \sigma\left(E_{j}\right) \delta_{1}^{2} \cdot \exp \left[-\frac{1}{2 \lambda_{1}^{2}}\left(E_{i}-E_{j}\right)^{2}\right] \\
& +\delta_{2}^{2} \cdot \alpha \frac{\mathrm{d} \sigma}{\mathrm{d} E}\left(E_{i}\right) \cdot \alpha \frac{\mathrm{d} \sigma}{\mathrm{d} E}\left(E_{j}\right) \cdot \exp \left[-\frac{1}{2 \lambda_{2}^{2}}\left(E_{i}-E_{j}\right)^{2}\right] .
\end{aligned}
$$


The first term is equivalent to the expression introduced by Schnabel [1] for smooth cross sections, while the second one is sensitive to energy regions with strongly varying cross sections. The quantity $\alpha$ can be considered as mean widths of the resonances, $\lambda_{2}$ as correlation length between the resonances and $\delta_{2}$ as a relative uncertainty with respect to the resonance height. Usually relative high values are chosen for $\delta_{2}$ because a slight change in the peak position implies a great change in the magnitude of the cross section.

\subsection{Implementation of Formalism}

In principle a Bayesian evaluation can be performed either by Monte Carlo techniques or in case of normal distribution by Generalized least Square (GLS) method. In this contribution we restrict ourselves to multivariate normal distributions and make use of the modified GLStechnique [1]. Thus we obtain expressions for the Bayesian update of the mean model cross sections $\vec{\sigma}_{1}$, the mean model error $\vec{\epsilon}_{\text {mod }}$ and the associated covariance matrices $\mathbf{A}_{1}$ and $\mathbf{K}_{1}$,

$$
\begin{aligned}
& \vec{\sigma}_{1}=\vec{\sigma}_{0}+\mathbf{A}_{0} \mathbf{S}^{T}\left(\mathbf{S} \mathbf{A}_{0} \mathbf{S}^{T}+\mathbf{S} \mathbf{K}_{0} \mathbf{S}^{T}+\mathbf{B}\right)^{-1}\left(\vec{\sigma}_{\exp }-\mathbf{S} \vec{\sigma}_{M}\right), \\
& \vec{\epsilon}_{1}=\mathbf{K}_{0} \mathbf{S}^{T}\left(\mathbf{S} \mathbf{A}_{0} \mathbf{S}^{T}+\mathbf{S} \mathbf{K}_{0} \mathbf{S}^{T}+\mathbf{B}\right)^{-1}\left(\vec{\sigma}_{\exp }-\mathbf{S} \vec{\sigma}_{M}\right), \\
& \mathbf{A}_{1}=\mathbf{A}_{0}-\mathbf{A}_{0} \mathbf{S}^{T}\left(\mathbf{S} \mathbf{A}_{0} \mathbf{S}^{T}+\mathbf{S} \mathbf{K}_{0} \mathbf{S}^{T}+\mathbf{B}\right)^{-1} \mathbf{S} \mathbf{A}_{0} \\
& \mathbf{K}_{1}=\mathbf{K}_{0}-\mathbf{K}_{0} \mathbf{S}^{T}\left(\mathbf{S} \mathbf{A}_{0} \mathbf{S}^{T}+\mathbf{S} \mathbf{K}_{0} \mathbf{S}^{T}+\mathbf{B}\right)^{-1} \mathbf{S} \mathbf{K}_{0} .
\end{aligned}
$$

where the matrix $\mathbf{S}$ provides the transformation from the model grid to the experimental grid. For simplicity we assumed in Eqs. (5) to (8) that the model grid contains all energies for which experimental data are given.

Due to the ansatz (2) the evaluated cross section is not $\vec{\sigma}_{1}$ but $\vec{\sigma}_{\text {true }}=\vec{\sigma}_{1}+\vec{\epsilon}_{1}$ and the corresponding covariance matrix

$$
\mathbf{U}_{1}=\mathbf{U}_{0}-\mathbf{C}_{0}\left(\mathbf{S} \mathbf{A}_{0} \mathbf{S}^{T}+\mathbf{S K}_{0} \mathbf{S}^{T}+\mathbf{B}\right)^{-1} \mathbf{C}_{0}^{T}
$$

with $\mathbf{U}_{0}=\mathbf{S} \mathbf{A}_{0} \mathbf{S}^{T}+\mathbf{K}_{0}$ and $\mathbf{C}_{0}=\mathbf{A}_{0} \mathbf{S}^{T}+\mathbf{K}_{0} \mathbf{S}^{T}$.

\section{Application}

In order to demonstrate the applicability of this formalism in the resonance range we apply the procedure on a schematic set of experimental data with known model defects. The example and the obtained results are discussed in detail in the following subsections.

\subsection{Generation of schematic example}

We start from a schematic R-matrix example of neutron $-{ }_{8}^{16} \mathrm{O}$ scattering including all partial waves up to $J=5 / 2$. In three partial waves, i.e. $J^{\pi}=1 / 2^{+}, 3 / 2^{+}$and $5 / 2^{-}$, we assume the existence of one pole leading to the single-channel R-matrix

$$
R^{J \pi}(E)=\frac{\gamma_{i}^{2}}{E_{i}-E} \text { for } \quad J^{\pi}=1 / 2^{+}, 3 / 2^{+} \text {and } 5 / 2^{+}
$$

and vanishing in all other partial waves. The chosen pole energies and widths are given in Tab 1. Using the R-matrix method the cross section $\sigma_{\text {calc }}$ was determined for the energies between $0.005 \mathrm{MeV}$ and $2.550 \mathrm{MeV}$ with an increment of $0.005 \mathrm{MeV}$ equivalent to a model grid of $N=510$ mesh points. 
Table 1. Comparison of the originally chosen pole parameters of the R-matrix calculation (left) and the parameter values obtained by fit of the experimental data distorted by model defects.

\begin{tabular}{llll||ll}
\hline & & chosen & pole values & fitted & pole values \\
$J^{\pi}$ & $i$ & $E_{i}[\mathrm{MeV}]$ & $\gamma_{i}[\mathrm{MeV}]$ & $E_{i}[\mathrm{MeV}]$ & $\gamma_{i}[\mathrm{MeV}]$ \\
\hline $1 / 2^{+}$ & 1 & 0.70 & 0.20 & 0.685905 & 0.195017 \\
$3 / 2^{+}$ & 2 & 1.20 & 0.40 & 1.150608 & 0.407358 \\
$5 / 2^{-}$ & 3 & 2.00 & 0.30 & 1.911950 & 0.355688 \\
\hline
\end{tabular}

In order to simulate the model defect we determine the mean value of the experimental data by applying a distortion function

$$
\bar{\sigma}_{\exp }(E)=\left[1+\alpha_{d} \frac{d \sigma_{\text {calc }}}{d E}(E)\right] \sigma_{\text {calc }}(E)
$$

with $\alpha_{d}=7 \times 10^{-6} \mathrm{MeV} / \mathrm{mbarn}$. Choosing a subset $\left\{E_{1}, E_{2}, \ldots, E_{M}\right\}$ of the $N$ mesh points we generate a set of schematic experimental data with a statistical uncertainty $\epsilon_{S}=0.03$ and a normalisation error of $\epsilon_{N}=0.03$ by

$$
\sigma_{\exp }\left(E_{j}\right)=\left(1+\epsilon_{N}\right) \bar{\sigma}_{\exp }\left(E_{j}\right) \cdot \mathcal{N}\left(1, \epsilon_{S}^{2}\right),
$$

where $\mathcal{N}\left(1, \epsilon_{S}^{2}\right)$ refers to a normal distribution with mean value 1 and the standard deviation $\epsilon_{S}$. Thus we are able to construct the experimental vector $\vec{\sigma}_{\text {exp }}$ which is of dimension $M$ and the $M \times M$ covariance matrix $\mathbf{B}$ of experimental cross sections,

$$
\mathbf{B}=\left(\begin{array}{cccc}
\left(\bar{\sigma}_{1}^{\exp }\right)^{2}\left[\epsilon_{s}^{2}+\epsilon_{N}^{2}\right] & \bar{\sigma}_{1}^{\exp } \bar{\sigma}_{2}^{\exp } \epsilon_{N}^{2} & \cdots & \bar{\sigma}_{1}^{\exp } \bar{\sigma}_{M}^{\exp } \epsilon_{N}^{2} \\
\bar{\sigma}_{2}^{\exp } \bar{\sigma}_{1}^{\exp } \epsilon_{N}^{2} & \left(\bar{\sigma}_{2}^{\exp }\right)^{2}\left[\epsilon_{s}^{2}+\epsilon_{N}^{2}\right] & \cdots & \bar{\sigma}_{2}^{\exp } \bar{\sigma}_{M}^{\exp } \epsilon_{N}^{2} \\
\vdots & \cdots & \ddots & \vdots \\
\bar{\sigma}_{M}^{\exp } \bar{\sigma}_{1}^{\exp } \epsilon_{N}^{2} & \bar{\sigma}_{L}^{\exp } \bar{\sigma}_{2}^{\exp } \epsilon_{N}^{2} & \cdots & \left(\bar{\sigma}_{M}^{\exp }\right)^{2}\left[\epsilon_{S}^{2}+\epsilon_{N}^{2}\right]
\end{array}\right)
$$

where $\bar{\sigma}_{j}^{\exp }=\bar{\sigma}_{\exp }\left(E_{j}\right)$.

In Fig. 1 a comparison of $\sigma_{\text {calc }}$ and $\sigma_{\text {exp }}$ at $M$ mesh points is given.
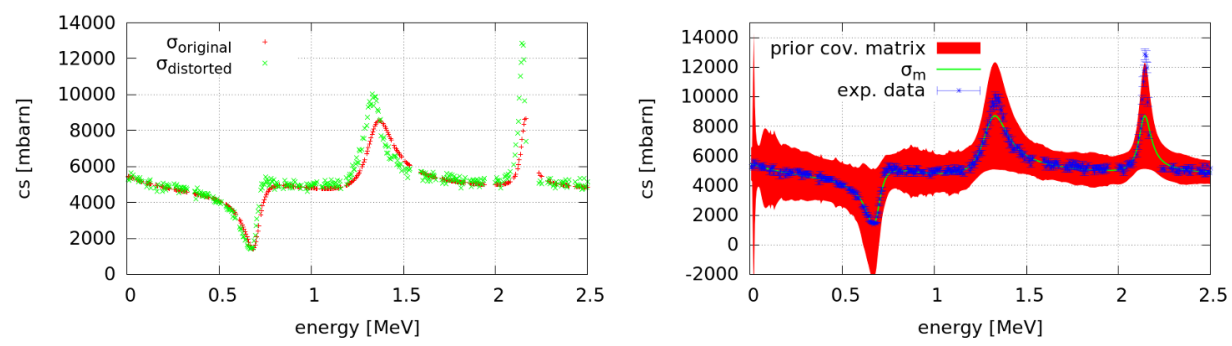

Figure 1. Left: Comparison of original and distorted data with $\alpha_{d}=7 \times 10^{-6}$. Right: schematic experimental data and best parameter cross section $\sigma_{M}(E)$ with $a$-priori variance $\sqrt{A_{0}\left(E_{j}, E_{j}\right)}$.

\subsection{Generation of prior}

The prior consists of the $a$-priori mean value of the cross section and the associated covariance matrix $\mathbf{A}_{0}$. In order to obtain the mean value we make a fit of the schematic experimental data 
with the R-matrix method. The best set of resonance parameters obtained from the schematic experimental data is given in Tab. 1 and yields the cross section $\sigma_{M}(E)$ displayed in the right part of Fig. 1.

In the next step, we assume an uncertainty width of $50 \%$ for each of the 6 best resonance parameters and perform $n=200$ calculations of the cross sections at all mesh points with different randomly chosen combinations of resonance parameters. Following [6] we are thus able to generate the $a$-priori covariance matrix $\mathbf{A}_{0}$ from the $n$ Monte Carlo sweeps. Using $\sigma_{M}(E)$ and the parameter values $\delta_{1}=0.05, \lambda_{1}=0.5 \mathrm{MeV}, \delta_{2}=1.50, \alpha=0.2 \mathrm{MeV}$ and $\lambda_{2}=0.10 \mathrm{MeV}$ in Eq. (4) yields the $a$-priori covariance matrix $\mathbf{K}_{0}$ of the model defect $\epsilon_{\bmod }$.

\subsection{Bayesian update and results}

In the previous subsection we described the generation of the schematic experimental data as well as the determination of the required $a$-priori information. Thus we are able to apply the Bayesian update procedure outlined in Subsect. 2.3. At this point it should be remarked that we use a surrogate model which means that we calculate our observables $\vec{\sigma}_{M}$ by the R-matrix method on the $N$ energies of the evaluation mesh and use these observables as parameters of the model. Hence, every point on the mesh is a random variable, but cannot be arbitrarily varied because the properties of the model enter via the $a$-priori covariance matrix $\mathbf{A}_{0}$.

The only quantity we still need for the evaluation is the matrix $\mathbf{S}$ which transforms from the grid of the evaluation to the grid of energies where experimental data are given. The matrix is of dimension $M \times N$ and is particularly simple in our example, i.e. it has only nonvanishing elements $\left(S_{i j}=1\right)$ on energy points where both calculated and experimental data are available.

With this preparation the Bayesian evaluation of the set of schematic experimental data is straightforward and allows us to study the differences due to the inclusion of model defects. A comparison of the evaluation without and with inclusion of model defects is shown in Fig. 2. The evaluation without model defects reproduces the experimental data quite well in regions of smooth energy dependence. However, the evaluation becomes problematic at resonances which can be best identified in the third resonance at $2.14 \mathrm{MeV}$. The model can never reach the height of the experimental peak (Fig. 3). If we account for model defects the agreement of the evaluated resonance cross sections with the experimental data (Figs. 2 and 3) significantly improves.

The comparison between the built-in model defect $\sigma_{\exp }-\sigma_{M}$ and the evaluated mean model defect $\epsilon_{\text {mod }}$ in Fig. 4 shows a striking similarity in size and structure. This result is very promising because it indicates that the procedure is indeed capable to extract the proper size and energy dependent structure of model defects.
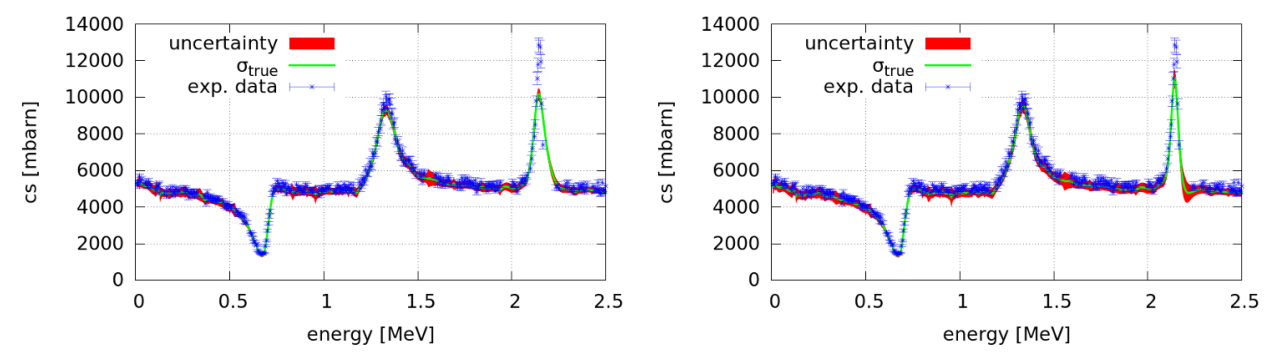

Figure 2. The true cross section $\sigma_{\text {true }}$ evaluated without (left) and with (right) model defects. 


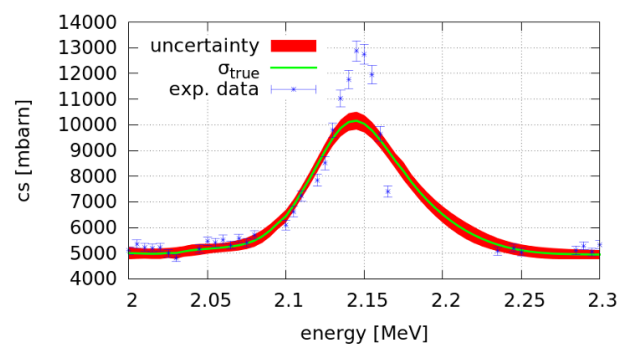

(a) Without model defects

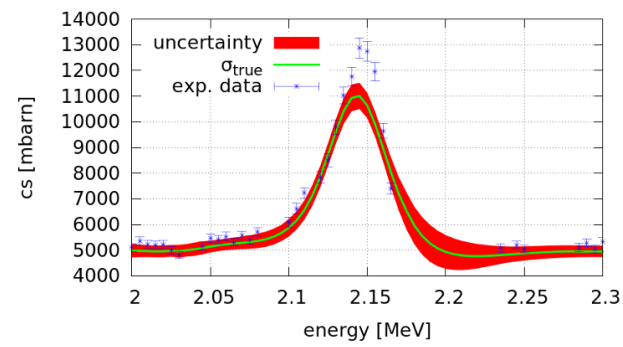

(b) With model defects

Figure 3. Comparison of the third resonance, evaluated without (left) and with (right) model defects.

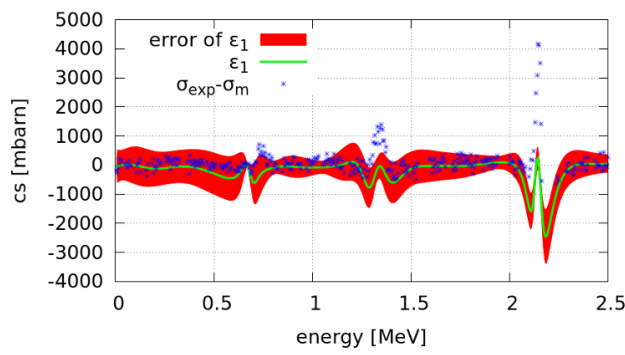

Figure 4. Comparison of the difference $\sigma_{\exp }-\sigma_{M}$ with the calculated model error $\epsilon_{1}$.

In Fig. 5 we consider the correlation matrices $\mathbf{A}_{1}$ updated without and with model defects. At first glance the correlations seem to be quite similar apart from the occurrence of anticorrelations in the presence of model defects. The size of the relative uncertainties differs

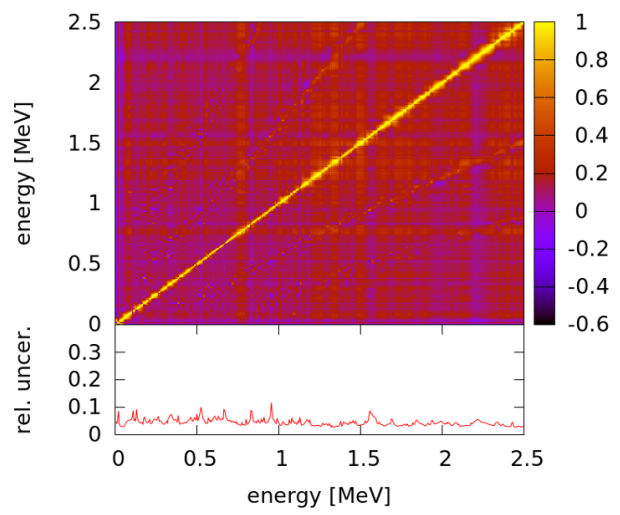

(a) Without model defects

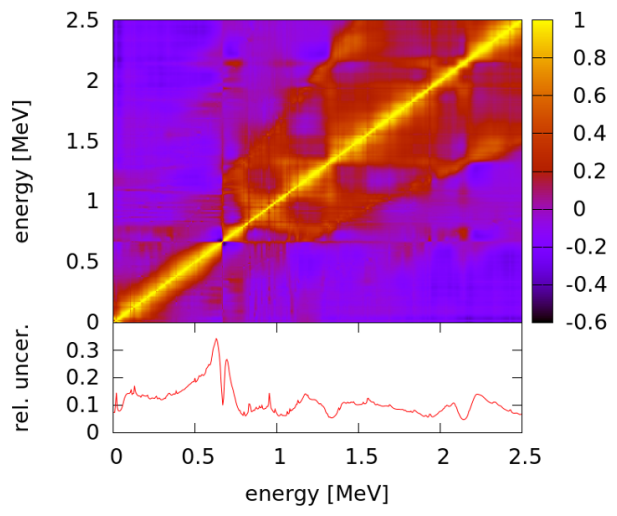

(b) With model defects

Figure 5. Correlation associated to matrix $\mathbf{A}_{1}$ evaluated without (left) and with (right) model defects. In the inset below the relative uncertainty $\sqrt{\mathbf{A}_{1}\left(E_{i}, E_{i}\right)} / \sigma_{\text {true }}$ is given. 
significantly and confirms the general finding that uncertainties tend to be underestimated if model defects are neglected in the evaluation.

In order to complete the comparison we show in Fig. 6 the correlation matrix $\mathbf{U}_{1}$ of the true cross section $\sigma_{\text {true }}$ which is the final result which should enter in an evaluated data file. In addition the updated covariance matrix $\mathbf{K}_{1}$ of the model error $\epsilon_{\bmod }$ is also shown in Fig. 6.


Figure 6. Correlation matrices associated to $\mathbf{U}_{1}$ (left) and $\mathbf{K}_{1}$ (right) with their relative uncertainties $\sqrt{\mathbf{U}_{1}\left(E_{i}, E_{i}\right)} / \sigma_{\text {true }}$ and $\sqrt{\mathbf{K}_{1}\left(E_{i}, E_{i}\right)} / \sigma_{\text {true }}$.

\section{Summary and Outlook}

We extended the recently developed formalism for model defects based on Gaussian processes $[1,5]$ to the resonance region. Key of the extension is the proposal of an alternative prior covariance matrix $\mathbf{K}_{0}$ of model defects, Eq. (4), which is primarily sensitive to the shape of resonances. This covariance matrix allows a Bayesian evaluation including model defects for the total energy range in a unified scheme simultaneously including the resonant and the smooth behavior of cross sections at low and intermediate projectile energies, respectively. The method also provides the magnitude of uncertainties as well as the associated covariance matrices including cross correlations between the different contributions.

\section{References}

[1] G. Schnabel, Large Scale Bayesian Nuclear Data Evaluation with Consistent Model Defects, PhD thesis, TU Wien (2015)

[2] P. Helgesson, H. Sjöstrand, Rev. Sci. Instr. 88, 115114 (2017)

[3] H. Leeb, D. Neudecker, Th. Srdinko, Nucl. Data Sheets 109, 2762 (2009)

[4] D. Neudecker, R. Capote, H. Leeb, Nucl. Instr. Meth. A 723, 163 (2013)

[5] G. Schnabel, H. Leeb, EPJ Web of Conferences 111, 09001 (2016)

[6] G. Schnabel, H. Leeb, Nucl. Instr. Method A 841, 87 (2017) 